\title{
Virtual International Authority File (VIAF)
}

Pollin, Christopher; christopher.pollin@uni-graz.at

VIAF ist eine virtuelle, internationale Normdatei und bietet einen Dienst an, der es Bibliotheken ermöglichen soll, abgeglichene, verlinkte und geclusterte Normdaten zu verwenden. Als Normdaten werden standardisierte und eindeutig zuzuordnende Einträge für bestimmte Gegenstandsbereiche verstanden.

Normdaten in VIAF werden von nationalen Partnern aggregiert, wie etwa der Gemeinsamen Normdatenbank (GND) in Deutschland, und erlauben es, Personen, Organisationen, Werke oder Orte zu identifizieren. So entsteht ein zusammengeführter Datensatz inklusive einer Konkordanzdatei von jeder erfassten Entität in VIAF. Dieser steht für Recherchen und Datenaustausch zur Verfügung. Zusätzlich werden weiterführende Informationen zu den einzelnen Entitäten mit angeboten.

Das Protocol for Metadata Harvesting der Open Archives Initiative (OAI$\mathrm{PMH}$ ) wird zur Aktualisierung der Datenbestände in VIAF verwendet. Monatlich findet ein Musterabgleich, ein sogenanntes 'Pattern Matching', statt. Dabei werden neue Datensätze mit den bestehenden zusammengeführt, um sicherzustellen, dass Dubletten aufgelöst werden. Als Identifikator fungiert eine eigene Normdatennummer in Form eines URI. Diese können somit auch als Referenzpunkt für Linked Open Data genutzt werden.

So repräsentiert die URI https://viaf.org/viaf/19685936 die Person Stefan Zweig und gleichzeitig ein Dokument im Web, das Information über Stefan Zweig anbietet.

\section{Literatur:}

- Bennett, Rick; Hengel-Dittrich, Christina; O'Neill, Edward T.; Tillett, Barbara B.: VIAF (Virtual International Authority File): Linking Die Deutsche Bibliothek and Library of Congress Name Authority Files. In: World Library and Information Congress: 72nd IFLA General Conference and Coucil. Seoul: 2006.

- Jannidis, Fotis; Kohle, Hubertus: Digital Humanities. Eine Einführung. Mit Abbildungen und Grafiken Digital Humanities. Hrsg. von und Malte Rehbein. Stuttgart: 2017.

- OCLC: VIAF. URL: https://www.oclc.org/de/viaf.html

\section{Verweise:}

Normdaten, Semantic Web, GND, GeoNames, Wikidata, Getty, Metadata Har- 
vesting

\section{Projekte:}

\section{VIAF}

\section{Themen:}

Archivierung

\section{Lexika}

- Edlex: Editionslexikon

\section{Zitiervorschlag:}

Pollin, Christopher. 2021. Virtual International Authority File (VIAF). In: KONDE Weißbuch. Hrsg. v. Helmut W. Klug unter Mitarbeit von Selina Galka und Elisabeth Steiner im HRSM Projekt "Kompetenznetzwerk Digitale Edition". URL: https://gams.uni-graz.at/o:konde.111 